GENE EXPRESSION

\title{
Controls and roles for trans-splicing
}

A few examples of trans-spliced RNAs - RNA sequences ligated from separate transcripts - have been experimentally validated and functionally characterized in various species. Trans-spliced RNAs are often inferred from transcriptome sequencing data sets, but dissecting the genuine trans-spliced RNAs from false positives resulting from technical artefacts remains a challenge. A new study reports a pipeline for prioritizing candidate trans-spliced RNAs from transcriptome sequencing data and uses this approach to identify a role for a trans-spliced non-coding RNA (ncRNA) in maintaining pluripotency in human cells.

Wu et al. carried out both long-read and short-read high-throughput transcriptome sequencing on the $\mathrm{H} 9$ human embryonic stem cell (hESC) line, and used equivalent publicly available sequencing data for the $\mathrm{H} 1 \mathrm{hESC}$ line. By aligning the long-read sequences to the reference human genome sequence, they identified 8,822 candidate trans-spliced RNAs. They then applied various bioinformatic filters to remove probable false positives, such as candidates that were not supported by short-read sequencing and by both cell lines, and those candidates with sequence features that were suggestive of artefactual generation. These filters eliminated $\sim 99.9 \%$ of candidates; of the remaining nine high-priority candidates, four passed subsequent experimental validation.

All four validated trans-splicing events were intragenic; that is, they resulted in a mis-ordered arrangement of exons from the same gene. Sequencing and experimental data indicate that this splicing occurred through trans-splicing between two copies of the primary transcript rather than through, for example, intramolecular splicing to generate a circular mRNA.

Three of the trans-splicing events brought an alternative sequence upstream of a full-length coding region. These events were associated with a different expression pattern relative to the regular, colinear isoforms across a panel of differentiated cell types.

Interestingly, the fourth trans-spliced RNA was a novel ncRNA, termed tsRMST. This ncRNA was found to be expressed at higher levels in pluripotent cells (both hESCs and human induced pluripotent stem cells (hiPSCs)) relative to differentiated cells. In support of a role for tsRMST in pluripotency, manipulation of tsRMST levels in hESCs showed that this ncRNA suppresses the expression of differentiation-associated genes. This function is mediated in part by recruiting the transcription factor NANOG and the repressive Polycomb protein SUZ12 to the promoters of these lineage-specific genes.

It will be interesting to use prioritization strategies in other cell types to determine how widespread trans-spliced RNAs are, and the scope of their biological functions.

Darren J. Burgess

ORIGINAL RESEARCH PAPER Wu, C. S. et al. Integrative transcriptome sequencing identifies trans-splicing events with important roles in

human embryonic stem cell pluripotency.

Genome Res. http://www.dx.doi.org/10.1101/ gr.159483.113 (2013) 